INTERVENTIONAL CARDIOLOGY AND SURGERY

\title{
Cost effectiveness of drug eluting coronary artery stenting in a UK setting: cost-utility study
}

A Bagust, A D Grayson, N D Palmer, R A Perry, T Walley

Heart 2006;92:68-74. doi: 10.1136/hrt.2004.053850

See end of article for authors' affiliations

Correspondence to:

Professor Adrian Bagust,

University of Liverpool Management School,

Chatham Street, Liverpoo

L69 7ZH, UK:

A.Bagusł@liv.ac.uk

Accepted 21 March 2005

Published Online First

12 May 2005
Objective: To assess the cost effectiveness of drug eluting stents (DES) compared with conventional stents for treatment of symptomatic coronary artery disease in the UK.

Design: Cost-utility analysis of audit based patient subgroups by means of a simple economic model.

Setting: Tertiary care.

Participants: 12 month audit data for 2884 patients receiving percutaneous coronary intervention with stenting at the Cardiothoracic Centre Liverpool between January 2000 and December 2002.

Main outcome measures: Risk of repeat revascularisation within 12 months of index procedure and reduction in risk from use of DES. Economic modelling was used to estimate the cost-utility ratio and threshold price premium.

Results: Four factors were identified for patients undergoing elective surgery $(\mathrm{n}=1951)$ and two for nonelective surgery $(n=933)$ to predict risk of repeat revascularisation within 12 months. Most patients fell within the subgroup with lowest risk (57\% of the elective surgery group with $5.6 \%$ risk and $91 \%$ of the nonelective surgery group with $9.9 \%$ risk). Modelled cost-utility ratios were acceptable for only one group of high risk patients undergoing non-elective surgery (only one patient in audit data). Restricting the number of DES for each patient improved results marginally: $4 \%$ of stents could then be drug eluting on economic grounds. The threshold price premium justifying $90 \%$ substitution of conventional stents was estimated to be $£ 112$ (US\$212, €162) (sirolimus stents) or £89 (US\$167, €130) (paclitaxel stents).

Conclusions: At current UK prices, DES are not cost effective compared with conventional stents except for a small minority of patients. Although the technology is clearly effective, general substitution is not justified unless the price premium falls substantially.
$\mathrm{P}$

ercutaneous coronary transluminal angioplasty (PTCA) is widely used to treat angina, but its effectiveness is limited by high rates of restenosis necessitating repeat intervention. The development of bare metal stents (BMS) sharply reduced but did not eliminate this limit. ${ }^{1}$ Restenosis from neointimal proliferation around BMS requires a further procedure in $14-25 \%$ of patients. ${ }^{2}$ Drug eluting stents (DES) release an antiproliferative agent to inhibit neointimal hyperplasia, minimising the risk and degree of restenosis, without systemic toxicity. ${ }^{3}{ }^{4}$ Three recent meta-analyses ${ }^{5-7}$ showed that DES prevented much of the restenosis and need for revascularisation still evident with BMS. However, no differences were found in the risk of mortality, stroke, or acute myocardial infarction (although studies to date had only limited power to detect such differences). This new technology is considerably more expensive (around $£ 500$ extra for each stent), obliging health care funding agencies to question how far use of DES should be supported. The National Institute for Health and Clinical Excellence issued revised guidance in $2003^{8}$ limiting National Health Service (NHS) use to a few subgroups of patients identified from clinical trials. ${ }^{9}$

This study considered the cost-utility of DES compared with BMS for treatment of symptomatic coronary artery disease from the perspective of NHS commissioners, based on audit data from one region of England. Patients may benefit to different degrees depending on their risk of restenosis and, therefore, we distinguished between subgroups of patients wherever possible to help target patients most likely to benefit from DES.

\section{METHODS}

We analysed cost-utility for the 12 months after the index intervention, when most repeat interventions due to rest- enosis would be expected, ${ }^{10}$ assuming that all later events would arise from progressive coronary heart disease. We assumed that the benefits of DES were confined to reduction in angina and the need for revascularisation procedures as current trial evidence suggests. For this short time period, discounting of costs and outcomes is unnecessary. Results are presented in the usual way as an incremental cost effectiveness ratio (ICER) for each device in different subgroups of patients.

\section{Data collection}

\section{DES effectiveness}

The two most widely used types of DES were selected for comparison with BMS: one coated with sirolimus (Cypher; Cordis Corporation, Miami, Florida, USA) and one with paclitaxel (Taxus, slow release model; Boston Scientific, Natick, Massachusetts, USA). Most stent trials have not reported the most important outcome for patients, practising clinicians, and health care funders: the risk of any repeat revascularisation (irrespective of lesion or vessel involved). Instead publications report angiographic restenoses (not all clinically significant) and event rates specific to the lesion or vessel initially revascularised. This selective reporting omits

\footnotetext{
Abbreviations: ARTS, arterial revascularisation therapies study; BMS, bare metal stents; CABG, coronary artery bypass grafting; CTC, Cardiothoracic Centre; DES, drug eluting stents; ICER, incremental cost effectiveness ratio; PTCA, percutaneous transluminal coronary angioplasty; QALY, quality adjusted life year; RAVEL, randomised study with the sirolimus eluting Velocity balloon expandable stent in the treatment of patients with de novo native coronary artery lesions; SIRIUS, sirolimus eluting balloon expandable stent in the treatment of patients with de novo native coronary artery lesions; SoS, stent or surgery; TAXUS, treatment of de novo coronary disease using a single paclitaxel eluting stent; TVR, target vessel revascularisation
} 
other interventions and exaggerates the apparent benefit attributable to DES. Most trials also involve protocol driven follow up angiography, which overstates both the risk of recurrence and the benefit of using DES. ${ }^{11}$ To estimate the efficacy of DES, we used target vessel revascularisation (TVR) as a proxy for overall revascularisation, since it is widely reported, but these estimates are necessarily optimistic in favour of DES.

For sirolimus based DES, two trials reported outcomes at 12 months follow up: RAVEL (randomised study with the sirolimus eluting Velocity balloon expandable stent in the treatment of patients with de novo native coronary artery lesions) $(\mathrm{n}=238)^{12}$ and SIRIUS (sirolimus eluting balloon expandable stent in the treatment of patients with de novo native coronary artery lesions) $(\mathrm{n}=1058) \cdot{ }^{13}$ Meta-analysis leads to TVR of $7.5 \%$ (Cypher) versus $24.9 \%$ (BMS), a relative risk reduction of $69.8 \%$ (95\% confidence interval $59.3 \%$ to $77.7 \%, \mathrm{p}<0.001)$. For paclitaxel based DES, 12 month TVR results from TAXUS (treatment of de novo coronary disease using a single paclitaxel eluting stent) I $(\mathrm{n}=60),{ }^{14}$ TAXUS II (slow release cohort, $\mathrm{n}=261),{ }^{15}$ and TAXUS IV $(\mathrm{n}=1314)^{16}$ gave overall TVR results of $7.3 \%$ (Taxus) versus $16.3 \%$ (BMS), a relative risk reduction of $55.3 \%(95 \%$ confidence interval $40.3 \%$ to $66.5 \%, \mathrm{p}<0.001$ ). Although the patient samples are not directly comparable, in the absence of any evidence to the contrary, we have assumed that the relative benefits of each DES are preserved despite differences in case mix across the studies. There is no direct comparative trial between sirolimus and paclitaxel based DES yet, but the results of the REALITY study are expected soon. We performed separate economic analyses for each.

\section{Patient subgroups}

To identify clinical or angiographic risk factors predisposing patients to subsequent revascularisation, we retrospectively analysed prospectively collected audit data from a consecutive series of 2884 patients ( 1951 for elective surgery, 933 for non-elective) undergoing stent placement at the Cardiothoracic Centre (CTC) in Liverpool between January 2000 and December 2002. All patients were followed up for 12 months. During this period CTC made minimal use of DES. We used data on patient demographics, heart disease

Table 1 Prevalence of risk factors of patients undergoing elective and non-elective stent placement

\begin{tabular}{lll}
\hline Patient risk factor & $\begin{array}{l}\text { Elective } \\
\text { (n=1951) }\end{array}$ & $\begin{array}{l}\text { Non-elective } \\
\text { (n=933) }\end{array}$ \\
\hline Age at intervention (years) & $60.3(53.6-67.4)$ & $61.2(53.4-69.4)$ \\
Women & $27.9 \%$ & $22.5 \% \%^{* *}$ \\
NYHA dyspnoea class $>$ II & $9.5 \%$ & $29.2 \%^{* *}$ \\
AMI & $3.9 \%$ & $39.4 \%^{* *}$ \\
Unstable angina & $6.7 \%$ & $55.2 \% * *$ \\
Cardiogenic shock & $0.0 \%$ & $2.1 \% \%^{* *}$ \\
Family history of CHD & $51.1 \%$ & $47.1 \% *$ \\
Hypercholesterolaemia & $85.3 \%$ & $84.6 \%$ \\
Hypertension & $49.5 \%$ & $42.4 \%^{* *}$ \\
Smoking history & $68.1 \%$ & $70.2 \%$ \\
Diabetes & $13.2 \%$ & $12.9 \%$ \\
Peripheral vascular disease & $4.4 \%$ & $4.1 \%$ \\
Cerebrovascular disease & $4.4 \%$ & $5.6 \%$ \\
Renal dysfunction & $1.0 \%$ & $0.9 \%$ \\
Respiratory disease & $5.6 \%$ & $6.3 \%$ \\
Ejection fraction $<50 \%$ & $16.6 \%$ & $32.1 \% \%^{* *}$ \\
Triple vessel disease & $12.0 \%$ & $16.0 \% * *$ \\
Left main stem $>50 \%$ & $1.3 \%$ & $1.8 \%$ \\
Prior CABG & $7.4 \%$ & $5.6 \%$ \\
\hline
\end{tabular}

${ }^{*} \mathrm{p}<0.05 ;{ }^{* *} \mathrm{p}<0.001$

AMI, acute myocardial infarction; $C A B G$, coronary artery bypass grafting; CHD, coronary heart disease; NYHA, New York Heart Association. severity, acuity, co-morbidity, and lesion characteristics in a proportional hazards regression analysis to isolate factors significantly and independently associated with increased risk of repeat revascularisation. We categorised subgroups by combinations of risk factors and calculated the absolute risk of reintervention for patients receiving BMS in each subgroup. Data were analysed with SAS version 8.2 (SAS Institute, Cary, North Carolina, USA) and SPSS version 10 (SPSS Inc, Chicago, Illinois, USA). The adequacy of proportional hazard models was confirmed by use of the "Cox with time dependent covariates" function.

Table 1 shows the prevalence of patient risk factors before intervention. Table 2 shows the frequency of lesion characteristics potentially indicating a high risk of revascularisation. Patients undergoing non-elective surgery were more likely to have had a recent acute myocardial infarction, unstable angina, or more severe disease (lower ejection fraction and higher New York Heart Association dyspnoea class) than patients having elective surgery.

\section{Valuation of outcomes}

Differences in health related utility (quality of life) between use of DES and BMS can arise in two ways: the patient may have recurrent severe symptoms while waiting for a repeat intervention (typically a few weeks) or may have pain and distress while undergoing the further intervention. The advantages of DES apply only to patients who would have had recurrent symptoms with BMS, so outcome gains from DES are proportional to the risk of requiring a repeat intervention.

Patient utilities (table 3) were estimated from EQ-5D results reported for the ARTS (arterial revascularisation therapies study) ${ }^{17}{ }^{18}$ and SoS (stent or surgery; R Stables, personal communication, 2003) trials. The difference in utility scores before and after treatment was combined with the duration of the disutility due to the intervention: one month for PTCA procedures but up to six months for coronary artery bypass grafting (CABG). Estimates for recurrent angina from ARTS and SoS strictly relate to patients with multivessel disease and may overstate the effect for patients with less complex disease treated percutaneously.

Table 2 Prevalence of lesion characteristics

\begin{tabular}{|c|c|c|}
\hline Lesion characteristic & $\begin{array}{l}\text { Elective } \\
(n=1951)\end{array}$ & $\begin{array}{l}\text { Non-elective } \\
(n=933)\end{array}$ \\
\hline Restenotic lesion & $2.7 \%$ & $1.2^{*}$ \\
\hline Graft lesion & $2.6 \%$ & $1.8 \%$ \\
\hline AHA C-type lesion & $43.3 \%$ & $39.0 \% *$ \\
\hline Thrombus & $4.3 \%$ & $33.2 \%$ ** \\
\hline Occlusion & $11.9 \%$ & $2.3 \%$ ** \\
\hline \multicolumn{3}{|l|}{ Length $(\mathrm{mm})$} \\
\hline 10-20 & $53.0 \%$ & $55.7 \% *$ \\
\hline$>20$ & $27.4 \%$ & $28.7 \%$ \\
\hline \multicolumn{3}{|l|}{ Tortuosity } \\
\hline $45-90^{\circ}$ & $33.0 \%$ & $25.6 \%$ ** \\
\hline$>90^{\circ}$ & $3.9 \%$ & $4.0 \%$ \\
\hline \multicolumn{3}{|l|}{ Angulation } \\
\hline $45-90^{\circ}$ & $25.9 \%$ & $17.6 \%$ ** \\
\hline$>90^{\circ}$ & $1.6 \%$ & $1.7 \%$ \\
\hline \multicolumn{3}{|l|}{ Diameter $(\mathrm{mm})$} \\
\hline$<2$ & $5.4 \%$ & $3.5 \%$ \\
\hline$>4$ & $9.5 \%$ & $8.8 \%$ \\
\hline Eccentric & $60.5 \%$ & $55.7 \% *$ \\
\hline Bifurcation & $17.8 \%$ & $14.7 \% *$ \\
\hline Calcification & $13.0 \%$ & $7.3 \%$ ** \\
\hline Multivessel PCI & $27.6 \%$ & $18.6 \% * *$ \\
\hline
\end{tabular}

${ }^{*} \mathrm{p}<0.05 ;{ }^{* *} \mathrm{p}<0.001$.

AHA, American Heart Association; $\mathrm{PCl}$, percutaneous coronary intervention. 
Table 3 Model parameters

\begin{tabular}{|c|c|c|}
\hline & Values (mean $(95 \% \mathrm{CI})$ ) & Source \\
\hline \multicolumn{3}{|l|}{ Resource use } \\
\hline \multicolumn{3}{|l|}{ Cardiology consultations } \\
\hline Elective index PTCA & $2.10(1.94$ to 2.26$)$ & CTC audit \\
\hline Non-elective index PTCA & $1.05(0.86$ to 1.24$)$ & \\
\hline Angiography for recurrent symptoms & 1.0 & Assumed in absence of reliable data \\
\hline \multicolumn{3}{|l|}{ Type of repeat procedure } \\
\hline \multicolumn{2}{|l|}{ After elective PTCA } & CTC audit \\
\hline Balloon angioplasty & $36.6 \%(28.9 \%$ to $45.0 \%)$ & \\
\hline Stented PTCA & $54.5 \%(46.0 \%$ to $62.7 \%)$ & \\
\hline CABG & $9.0 \%(5.1 \%$ to $15.2 \%)$ & \\
\hline \multicolumn{2}{|l|}{ After non-elective PTCA } & CTC audit \\
\hline Balloon angioplasty & $27.4 \%(19.0 \%$ to $37.6 \%)$ & \\
\hline Stented PTCA & $54.5 \%$ (44.2\% to $64.9 \%)$ & \\
\hline CABG & $17.9 \%$ (11.1\% to $27.4 \%)$ & \\
\hline \multicolumn{3}{|l|}{ Repeat PTCA stents used } \\
\hline Elective index PTCA & $1.87(1.62$ to 2.15$)$ & CTC audit \\
\hline Non-elective index PTCA & $1.71(1.50$ to 1.97$)$ & \\
\hline \multicolumn{3}{|l|}{ Follow up cardiology visits } \\
\hline Elective index PTCA & 2.18 (1.72 to 2.64$)$ & CTC audit \\
\hline Non-elective index PTCA & $1.80(1.45$ to 2.15$)$ & \\
\hline \multicolumn{3}{|l|}{ Follow up $C T$ surgery visits } \\
\hline Elective index PTCA & $0.81(0.42$ to 1.20$)$ & CTC audit \\
\hline Non-elective index PTCA & $0.48(0.23$ to 0.74$)$ & \\
\hline \multicolumn{3}{|l|}{ Unit costs (£) } \\
\hline \multicolumn{3}{|l|}{ Cardiology } \\
\hline First outpatient visit & 130.44 & NHS tariff costs $2004 / 5$ E15op deflated to $2003 / 4$ prices \\
\hline Follow up outpatient visit & 93.03 & NHS tariff costs 2004/5 E16op deflated to $2003 / 4$ prices \\
\hline Angiogram & 372.47 & NHS reference costs 2003 EO2op \\
\hline Elective PTCA & 3190.00 & NHS APC spell costs $2004 / 5$ E15 deflated to $2003 / 4$ prices \\
\hline Non-elective PTCA & 4179.00 & \\
\hline Elective CABG & 7750.00 & NHS APC Spell Costs 2004/5 E04 deflated to $2003 / 4$ prices \\
\hline Non-elective CABG & 9460.00 & \\
\hline \multicolumn{2}{|l|}{ Cardiac surgery } & $\begin{array}{l}\text { NHS reference costs spec } 170 \text { adjusted by tariff costs } \\
\text { cardiology ratios }\end{array}$ \\
\hline $\begin{array}{l}\text { First outpatient visit } \\
\text { Follow up outpatient visit }\end{array}$ & $\begin{array}{l}214.39 \\
171.67\end{array}$ & \\
\hline $\begin{array}{l}\text { Follow up outpatient visif } \\
\text { Cost per uncoated stent }\end{array}$ & 370.00 & Market average \\
\hline Price premium per drug eluting stent & 500.00 & List price difference (assumed baseline) \\
\hline \multicolumn{3}{|l|}{ Utility } \\
\hline Annual QALYs lost to angina & $0.135(0.122$ to 0.148$)$ & ARTS and SoS trials \\
\hline QALYs lost per PTCA & 0.0056 (0.0051 to 0.0062$)$ & \\
\hline QALYs lost per CABG & $0.033(0.031$ to 0.035$)$ & \\
\hline \multicolumn{3}{|l|}{ Waiting time for revascularisation (weeks) } \\
\hline For balloon angioplasty after elective index PTCA & $27.3(22.2$ to 32.4$)$ & CTC audit \\
\hline For stented PTCA/CABG after elective index PTCA & $15.0(12.1$ to 18.0$)$ & \\
\hline For balloon angioplasty after non-elective index PTCA & 19.5 (13.3 to 25.7$)$ & \\
\hline For stented PTCA/CABG after non-elective index PTCA & $14.0(9.6$ to 18.4$)$ & \\
\hline
\end{tabular}

\section{Costs and resource use}

Four revascularisation treatment costs were considered: specialist consultations for patients with recurrent symptoms; hospital investigations undertaken for these patients; repeat interventions undertaken (PTCA, stented percutaneous coronary intervention, or CABG); and specialist follow up (table 3). Where possible NHS reference costs for $2003^{19}$ were used (in UK£; $£ 1$ is approximately US\$1.8 and $€ 1.4$ ), supplemented as necessary by information from CTC. NHS tariff costs for 2004/5 were deflated to equivalent 2003 reference costs for similar admissions and procedures.

\section{Economic model}

Costs and outcomes over 12 months were compared between patients initially receiving BMS and those receiving DES. Cost-utility was assessed by comparing the net additional cost (that is, the extra cost for each DES used, less any savings in treatment costs) with the loss of utility avoided as a result of fewer reinterventions expected from use of DES (appendix 1). We modelled two purchasing policies: 100\% use of BMS versus $100 \%$ DES. We assumed that cardiologists do not mix stent types when treating a patient, since it is not clear from clinical evidence whether mixing stents may compromise the effectiveness of the more efficacious device. However, as the economic evaluation considers only incremental differences in costs, the results are unchanged if we consider only those BMS substituted by DES, as long as patient outcomes are identical. We also assumed that a patient undergoing a repeat intervention received the same stent as in the index procedure.

Initially we constructed a complex life table model ${ }^{9}$ but preliminary analysis of model results showed that many model variables were effectively redundant when we assumed that no gain can be attributed to DES in relation to mortality, stroke, and myocardial infarction. Therefore, we developed a simpler model with only those elements where DES is likely to generate differences.

\section{RESULTS}

\section{Patient subgroups}

Four clinical variables were found to be significant independent predictors of repeat revascularisation after elective surgery within 12 months, and only two for non-elective surgery (table 4). Table 5 compares the absolute risks for subgroups according to the number of these risk factors (elective from none (A) to three or four (D); non-elective for 
Table 4 Proportional hazard models for risk of repeat revascularisation within 12 months

\begin{tabular}{lll}
\hline Risk factor & HR & p Value \\
\hline Patients undergoing elective PCl $(n=1951)$ & & \\
$\quad$ Calcification & 1.89 & 0.002 \\
$\quad$ Angulation $>45^{\circ}$ & 1.51 & 0.019 \\
Restenotic lesion & 2.19 & 0.032 \\
$\quad$ Triple vessel disease & 1.56 & 0.042 \\
Patients undergoing non-elective PCl $(n=933)$ & & \\
$\quad$ Vessel diameter $<2 \mathrm{~mm}$ & 2.90 & 0.004 \\
$\quad$ Prior CABG & 2.27 & 0.015 \\
\hline HR, hazard ratio. & & \\
\hline
\end{tabular}

none $(\mathrm{X})$ to two $(\mathrm{Z})$ ). Most patients fell within the subgroups with the lowest risk (57\% of elective surgeries and $91 \%$ of non-elective surgeries). The risk of a second revascularisation within 12 months is the main variable driving the model's results.

\section{Elective treatment}

When we consider the average number of stents placed in each patient, none of the four elective subgroups has an ICER below the usually quoted UK cost effectiveness threshold ( $£ 30000$ per quality adjusted life year (QALY) gained). If a cardiologist is able to predict how many stents will be required to treat a patient, we can estimate the expected ICER for that number of stents (table 6). Cost effectiveness is achieved only when a single DES is implanted in a patient with two or more risk factors present.

\section{Non-elective treatment}

Only for the smallest group (Z, both risk factors present) are DES always cost effective (table 6). Where stent numbers can be predicted, use of a single DES is always cost effective if at least one risk factor is present, and up to two (paclitaxel) or three (sirolimus) DES can be justified if both risk factors apply.

\section{Price premium}

All analyses used a price premium per DES based on approximate UK list prices ( $£ 500$ more than BMS). In practice hospitals may be able to negotiate discounts. We estimated the additional price per stent that could be justified to achieve an ICER of $£ 30000$ or less per QALY gained or for cost neutrality after 12 months of follow up (table 6).

The relation between the DES price premium and what proportion of stents should be purchased as DES is useful for budget holders who may be able to negotiate a lower price per DES. Figure 1 displays cumulative proportions for both cost effectiveness and cost neutrality for sirolimus DES. For more than $50 \%$ usage of DES, the price premium should be less than $£ 221$ (cost effectiveness) or $£ 146$ (cost neutrality) and for $90 \%$ usage no more than $£ 112$ and $£ 80$, respectively. Equivalent price thresholds for paclitaxel DES are lower.

\section{Sensitivity and uncertainty}

The robustness of cost effectiveness results to parameter uncertainty was examined by one way sensitivity analysis (with 95\% confidence intervals) and combined extreme values analysis. Of the seven main patient groups, one (patients undergoing non-elective surgery with one risk factor, Y) was genuinely borderline, being sensitive to several model parameters, and one (elective surgery for patients with three or four risk factors, D) can become cost effective if very few stents are used. The extreme values analysis showed that altered decisions were possible in three high risk patient groups (D, Y, and Z), though for $99 \%$ of elective surgeries and $91 \%$ of non-elective surgeries the conclusions are robust.

Table 5 Absolute risk of repeat revascularisation in 12 months of follow up for risk subgroups

\begin{tabular}{|c|c|c|c|c|c|c|c|}
\hline \multicolumn{8}{|c|}{ Patients undergoing elective surgery } \\
\hline \multirow{2}{*}{$\begin{array}{l}\text { Number of risk } \\
\text { factors }\end{array}$} & \multicolumn{4}{|c|}{ Subgroup risk profile } & \multirow{2}{*}{$\begin{array}{l}\text { Absolute } \\
\text { risk (\%) }\end{array}$} & \multirow[b]{2}{*}{$95 \% \mathrm{Cl}$} & \multirow{2}{*}{$\begin{array}{l}\text { Proportion of } \\
\text { patients }\end{array}$} \\
\hline & Calcification & Angulation $>45^{\circ}$ & Restenotic lesion & Triple vessel disease & & & \\
\hline $0(A)$ & & & & & 5.6 & 4.3 to 6.9 & $57.2 \%$ \\
\hline $1(B)$ & & & & & 8.4 & 6.9 to 10.1 & $31.6 \%$ \\
\hline B1 & No & Yes & No & No & 7.7 & 5.4 to 10.2 & $17.7 \%$ \\
\hline B2 & No & No & No & Yes & 7.7 & 4.9 to 10.7 & $6.3 \%$ \\
\hline B3 & Yes & No & No & No & 10.5 & 7.2 to 14.1 & $6.1 \%$ \\
\hline B4 & No & No & Yes & No & 11.1 & 5.8 to 16.8 & $1.5 \%$ \\
\hline $2(C)$ & & & & & 16.6 & 14.4 to 18.8 & $10.1 \%$ \\
\hline $\mathrm{Cl}$ & No & Yes & No & Yes & 14.8 & 11.5 to 18.4 & $3.6 \%$ \\
\hline $\mathrm{C} 2$ & Yes & Yes & No & No & 17.4 & 13.8 to 21.4 & $4.8 \%$ \\
\hline $\mathrm{C} 3$ & Yes & No & No & Yes & 17.3 & 13.4 to 21.6 & $0.9 \%$ \\
\hline $\mathrm{C} 4$ & No & Yes & Yes & No & 17.9 & 12.9 to 23.7 & $0.3 \%$ \\
\hline $\mathrm{C} 5$ & No & No & Yes & Yes & 17.9 & 12.7 to 24.0 & $0.4 \%$ \\
\hline C6 & Yes & No & Yes & No & 20.4 & 15.0 to 26.4 & $0.2 \%$ \\
\hline 3 or 4 (D) & & & & & 24.6 & 21.5 to 27.9 & $1.1 \%$ \\
\hline D1 & Yes & Yes & No & Yes & 23.7 & 19.6 to 28.1 & $0.8 \%$ \\
\hline D2 & No & Yes & Yes & Yes & 24.2 & 18.9 to 30.1 & $0.1 \%$ \\
\hline D3 & Yes & Yes & Yes & No & 26.5 & 21.2 to 32.4 & $0.2 \%$ \\
\hline D4 & Yes & No & Yes & Yes & 26.5 & 21.0 to 32.5 & $0.0 \%$ \\
\hline D5 & Yes & Yes & Yes & Yes & 32.2 & 26.7 to 38.0 & $0.1 \%$ \\
\hline \multicolumn{8}{|c|}{ Patients undergoing non-elective surgery } \\
\hline & \multicolumn{4}{|c|}{ Subgroup risk profile } & \multirow{2}{*}{$\begin{array}{l}\text { Absolute } \\
\text { risk (\%) }\end{array}$} & \multirow[b]{2}{*}{$95 \% \mathrm{Cl}$} & \multirow{2}{*}{$\begin{array}{l}\text { Proportion of } \\
\text { patients }\end{array}$} \\
\hline & Vessel diame & $<2 \mathrm{~mm}$ & Prior CABG & & & & \\
\hline $0(X)$ & & & & & 9.9 & 6.9 to 10.8 & $91.0 \%$ \\
\hline $1(Y)$ & & & & & 22.2 & 15.5 to 29.6 & $8.9 \%$ \\
\hline Y1 & Yes & & No & & 25.3 & 13.8 to 36.8 & $3.4 \%$ \\
\hline Y2 & No & & Yes & & 20.3 & 11.2 to 29.4 & $5.5 \%$ \\
\hline $2(Z)$ & & & & & 40.4 & 29.4 to 51.9 & $0.1 \%$ \\
\hline
\end{tabular}


Table 6 Cost effectiveness of drug eluting versus bare metal stents (baseline) after 12 months of follow up

\begin{tabular}{|c|c|c|c|c|c|c|c|c|}
\hline & & \multicolumn{4}{|c|}{ Elective surgery risk groups } & \multicolumn{3}{|c|}{ Non-elective surgery risk groups } \\
\hline & & A & B & C & D & $\mathbf{x}$ & $\mathbf{Y}$ & z \\
\hline \multicolumn{9}{|c|}{ Incremental cost per QALY gained } \\
\hline Stent & No used & & & & & & & \\
\hline \multirow[t]{4}{*}{ Sirolimus } & 1 & $£ 144900$ & $£ 71500$ & $£ 0^{*}$ & $-£ 24000^{*}$ & $£ 68500$ & $-£ 25400^{*}$ & $-£ 54100^{*}$ \\
\hline & 2 & $£ 363400$ & $£ 216500$ & $£ 73600$ & $£ 25600$ & $£ 226000$ & $£ 38300$ & $-£ 19100^{*}$ \\
\hline & 3 & $£ 582000$ & $£ 361600$ & $£ 147200$ & $£ 75200$ & $£ 383500$ & $£ 102000$ & $£ 15800^{*}$ \\
\hline & Mean & $£ 238900$ & $£ 179700$ & $£ 85200$ & $£ 51600$ & $£ 133600$ & $£ 30600$ & $-£ 23700^{*}$ \\
\hline \multirow[t]{4}{*}{ Paclitaxel } & 1 & $£ 205800$ & $£ 113000$ & $£ 22700$ & $-£ 7700^{*}$ & $£ 113500$ & $£ 5100^{*}$ & $-£ 41400^{*}$ \\
\hline & 2 & $£ 481900$ & $£ 296300$ & $£ 115700$ & $£ 55000$ & $£ 312500$ & $£ 75400$ & $£ 2800^{*}$ \\
\hline & 3 & $£ 758100$ & $£ 479600$ & $£ 208700$ & $£ 117700$ & $£ 511600$ & $£ 155800$ & $£ 46900$ \\
\hline & Mean & $£ 324400$ & $£ 249700$ & $£ 130200$ & $£ 87900$ & $£ 195800$ & $£ 65700$ & $-£ 3000^{*}$ \\
\hline \multicolumn{9}{|c|}{ Maximum price premium per drug eluting stent } \\
\hline Stent & ICER & & & & & & & \\
\hline \multirow[t]{2}{*}{ Sirolimus } & $£ 30000$ & $£ 170$ & $£ 209$ & $£ 330$ & $£ 416$ & $£ 271$ & $£ 497$ & $£ 888$ \\
\hline & $£ 0$ & $£ 122$ & $£ 150$ & $£ 238$ & $£ 300$ & $£ 205$ & $£ 376$ & $£ 671$ \\
\hline \multirow[t]{2}{*}{ Paclitaxel } & $£ 30000$ & $£ 134$ & $£ 164$ & $£ 259$ & $£ 325$ & $£ 213$ & $£ 388$ & $£ 683$ \\
\hline & $£ 0$ & $£ 96$ & $£ 118$ & $£ 186$ & $£ 234$ & $£ 161$ & $£ 293$ & $£ 517$ \\
\hline
\end{tabular}

Sensitivity can be analysed only on quantifiable model parameters but this does not exhaust the potential sources of uncertainty, since many assumptions are required in model construction not amenable to enumeration. Appendix 2 summarises five key assumptions and their potential impact on cost effectiveness.

\section{DISCUSSION}

The main determinant of cost effectiveness is the price premium for DES compared with that for BMS. Assuming current unit pricing of DES in UK, the use of DES can be considered cost effective only for any patient with a 12 month risk of requiring a second intervention greater than $12 \%$ (sirolimus) or 15\% (paclitaxel) and only for elective surgery for a patient who can be treated with a single DES. Therefore, use of DES in the UK would be justifiable on economic grounds for only about $4 \%$ of patients, despite the evident effectiveness of DES in preventing restenosis. Thus, a technology that is clearly effective is rendered inappropriate to implement except in a very restrictive manner due to its current high price.

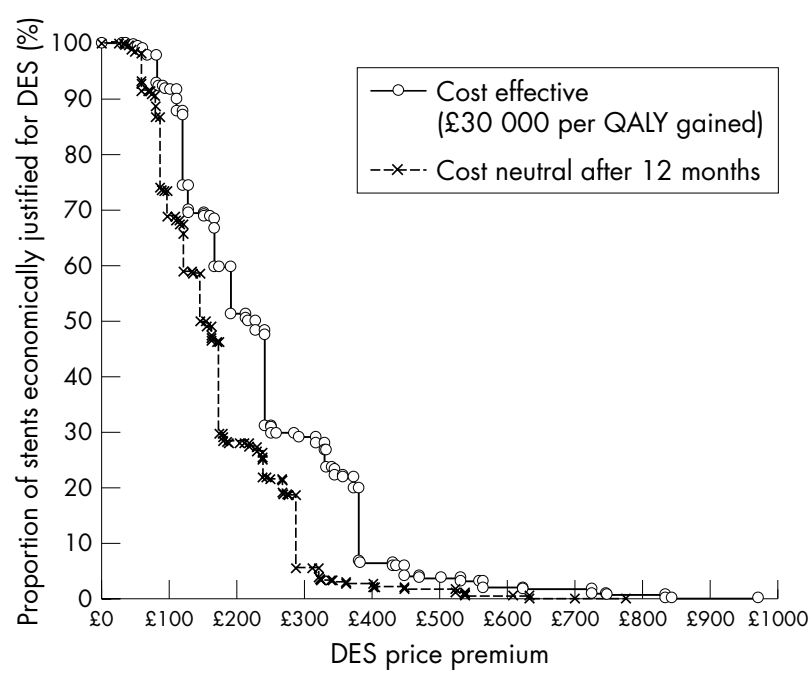

Figure 1 Proportion of stents used that satisfy economic criteria at different values of the drug eluting stent price premium for sirolimus coated stents.
The statistical models developed from CTC audit data indicate that the risk of repeat intervention for patients who had elective surgery is related to lesion characteristics and the extent of disease and after non-elective surgery to diseased narrow vessels and prior history of CABG. No traditional cardiac risk factors or co-morbidities (such as poor ventricular function, peripheral vascular disease, diabetes, renal disease, smoking, hypertension, and hypercholesterolaemia) were identified as increasing the need for a second intervention. This suggests that short term recurrent angina is largely a feature of vascular anatomy rather than of long term disease progression. A supplementary analysis of CTC patients followed up for 18 months comparing patients with versus patients without diabetes showed that survival curves diverged after 15 months, supporting the view that traditional risk factors act on longer timescales than do those associated with in-stent restenosis. In most patients, the risk of reintervention was lower then commonly understood (5.6\% after elective and 9.0\% after non-elective surgery), indicating that the need for improved stent performance may be limited to a few high risk patients.

Two other studies of the cost effectiveness of DES versus BMS have been published so far. A Spanish cost consequence study $^{8}$ based on a meta-analysis used a price per BMS of $€ 1000$ and per DES of $€ 2000$, concluding that DES would be cost neutral at a price of $€ 1448$. An American study based on SIRIUS and funded by the manufacturer of the Cypher stent reported an ICER of $\$ 27540$ per QALY. ${ }^{20}$ The results of the American study cannot be directly compared with our findings, being based on a single trial of uncomplicated planned interventions and employing utility gains assessed in a trial of emergency treatment for acute myocardial infarction. Neither study reflects real world NHS practice, nor UK NHS costs.

The main limitations of this study arise from lack of appropriate clinical trial results: small trials of insufficient duration to show differences in survival; bias from unblinded protocol angiography; and inadequate reporting of key outcomes. Any proven survival advantage would dramatically alter our findings, but thus far evidence is lacking. We therefore assumed that the full benefit of DES on angina and revascularisation will be seen within 12 months. ${ }^{12}$ Any subsequent added (or reduced) benefit can affect our results, though such effects would probably be much smaller than those included in the model. Also we have assumed that only one repeat procedure is allowed for each patient. Our earlier 
modelling suggested that $2-4 \%$ of patients may require a second reintervention but that the net effect on cost-utility results of this simplification is negligible.

The study is necessarily UK specific and our audit data are confined to a single centre serving a population of about 2.8 million. Risk estimates and factors may vary in other areas, though risks are unlikely to be underestimated, as north west England has a high standardised mortality ratio for heart disease. The CTC in Liverpool has been at the forefront in the UK for implementing a comprehensive cardiac interventions audit system and the quality of data is high. ${ }^{21}$

At present, therefore, we believe that the use of DES in preference to BMS should be restricted to high risk patients. New clinical evidence or a change in price may alter these conclusions.

\section{ACKNOWLEDGEMENTS}

We acknowledge the cooperation and assistance given to us by all consultant cardiologists at the CTC, by Mr A Beaumont of the CTC PCI registry, and by Dr R Hill (University of Liverpool) for bibliographical support.

\section{Authors' affiliations}

A Bagust, University of Liverpool Management School, Liverpool, UK A D Grayson, N D Palmer, R A Perry, Cardiothoracic Centre Liverpool NHS Trust, Liverpool, UK

T Walley, Prescribing Research Group, Department of Pharmacology and Therapeutics, University of Liverpool, Liverpool, UK

Funding: An early version of this work was funded as part of an appraisal on behalf of the National Institute for Clinical Excellence. $A B$ is partly funded by project funds from the NHS HTA programme. TW is Director of the NHS HTA programme. Neither NICE nor NCCHTA had any influence over the design, analysis, or reporting of this study.

\section{Competing interests: none}

Ethics approval: no separate ethics approval required.

Contributors: $A B$ carried out the economic analysis and drafted the paper. ADG extracted and analysed CTC audit data, carried out proportional hazards risk modelling, and revised the draft paper. TW conceived the study, contributed to its design, and participated in revising the draft paper. NP and RAP provided specialist cardiological advice to the study and participated in revising the draft paper. $A B$ is guarantor for the paper.

\section{APPENDIX 1}

\section{MODEL RELATIONS}

Incremental cost effectiveness ratio (ICER) $=$ incremental cost:incremental benefit where

Incremental cost per repeat intervention avoided

$=($ DES price premium $\times$ average number of stents per patient)/(risk of second procedure $\times$ DES relative risk reduction)

- average cost of re-referral and investigation per patient with recurrent symptoms

- average cost per patient undergoing repeat revascularisation procedure

- average cost per patient of additional post-revascularisation follow up care

and

Incremental benefit per repeat intervention avoided

$=$ annual QALY loss per year with severe angina $\times$ average time spent with symptoms (weeks)/52

+ average QALYs lost per patient recovering from repeat procedure

DES, drug eluting stent; QALY, quality adjusted life year.

\section{APPENDIX 2}

\section{NON-PARAMETRIC UNCERTAINTY}

\begin{tabular}{|c|c|c|}
\hline Uncertainty factor & Degree of uncertainty & Potential impact on cost effectiveness \\
\hline $\begin{array}{l}\text { Expected survival: we assume that DES } \\
\text { confers no advantage in lifetime survival } \\
\text { over bare metal stent }\end{array}$ & $\begin{array}{l}\text { No evidence of survival difference has been } \\
\text { detected in short term trials, but this may only } \\
\text { appear after follow up of } 10 \text { years or more }\end{array}$ & $\begin{array}{l}\text { Cost effectiveness analysis is based on very small utility } \\
\text { gains of short duration from impaired quality of life before } \\
\text { repeat intervention (equivalent to less than one month of } \\
\text { extended survival). Thus, any survival advantage would be } \\
\text { of greater effect and invalidate current model results }\end{array}$ \\
\hline $\begin{array}{l}\text { Trial protocol contamination: most trial } \\
\text { evidence is confounded by protocol } \\
\text { investigations that inflate repeat intervention } \\
\text { rates. Corrections for "clinically driven" } \\
\text { interventions are suspect as a posteriori } \\
\text { adjustments }\end{array}$ & $\begin{array}{l}\text { Comparison of trial subgroups with and without } \\
\text { trial angiography allow adjustments to be made } \\
\text { in some cases. It is not clear whether this } \\
\text { process is generalisable }\end{array}$ & $\begin{array}{l}\text { This inherent bias generally favours DES, since it inflates } \\
\text { the apparent recurrent risk amenable to improvement by } \\
\text { DES. This effect is largely offset by use of audit data for } \\
\text { patient risks and use of relative (not absolute) measures of } \\
\text { DES efficacy }\end{array}$ \\
\hline $\begin{array}{l}\text { Duration of effect: we assume that the } \\
\text { benefits of DES in avoiding restenotic } \\
\text { recurrence of symptoms is limited to } \\
12 \text { months. }\end{array}$ & $\begin{array}{l}\text { Assumption is consistent with trial and registry } \\
\text { evidence, but these are not of sufficient duration } \\
\text { to detect longer term differences. }\end{array}$ & $\begin{array}{l}\text { Since the outcome gains in the current cost effectiveness } \\
\text { analysis are very small, any additional benefit is likely to } \\
\text { change results significantly }\end{array}$ \\
\hline $\begin{array}{l}\text { Absence of true outcomes: most trials } \\
\text { report a variety of intermediate outcomes } \\
\text { and virtually none provide the total } \\
\text { number of repeat revascularisations }\end{array}$ & $\begin{array}{l}\text { For low risk patients, use of target vessel } \\
\text { revascularisation appears to be a reasonable } \\
\text { proxy for true outcomes. It is not clear whether } \\
\text { this assumption is valid for high risk groups }\end{array}$ & $\begin{array}{l}\text { Where additional interventions are omitted from both trial } \\
\text { arms, the apparent relative benefit of using DES is inflated } \\
\text { and this may have a large effect on cost effectiveness } \\
\text { results }\end{array}$ \\
\hline $\begin{array}{l}\text { Unit costs: the new NHS system of fixed } \\
\text { reimbursement costs applies to virtually } \\
\text { all the important resources in the model } \\
\text { and is due to be extended in future. We } \\
\text { therefore assume that from the perspective } \\
\text { of a service commissioner, there is no } \\
\text { uncertainty in unit costs }\end{array}$ & $\begin{array}{l}\text { NHS financial returns suggest large intersite } \\
\text { differences in unit costs (possibly due to local } \\
\text { application of accounting rules). It is not clear } \\
\text { how the new system will affect hospitals }\end{array}$ & $\begin{array}{l}\text { Sensitivity analysis of resource use suggests that cost } \\
\text { effectiveness results are vulnerable to unit cost changes in } \\
\text { only one area: the relative cost of coronary artery bypass } \\
\text { grafting episodes of care compared with percutaneous } \\
\text { transluminal angioplasty episodes }\end{array}$ \\
\hline
\end{tabular}




\section{REFERENCES}

1 Dundar Y, Hill RA, Bakhai A, et al. Angioplasty and stents in coronary artery disease: a systematic review and meta-analysis. Scand Cardiovasc J 2004:38:200-10.

2 British Cardiac Society, British Cardiovascular Intervention Society (BCIS) Submission document for NICE re-appraisal of coronary stenting 2002/3 2002.

3 Jenkins NP, Prendergast BD, Thomas $M$. Drug eluting stents. BMJ 2002;325:1315-6.

4 Fattori, Piva T. Drug-eluting stents in vascular intervention. Lancet 2003;361:247-9.

5 Hill R, Dundar Y, Bakhai A, et al. Drug-eluting stents: an early systematic review to inform policy. Eur Heart J 2004;25:902-19.

6 Oliva G, Espallargues M, Pons J. Antiproliferative drug-eluting stents: systematic review of the benefits and estimate of economic impact. Rev Esp Cardiol 2004;57:617-28

7 Babapulle M, Joseph L, Bélisle $P$, et al. A hierarchical Bayesian meta-analysis of randomised clinical trials of drug-eluting stents. Lancet 2004;364:583-91.

8 National Institute for Clinical Excellence. Guidance on the use of coronary artery stents. Technology appraisal guidance 71. London: NICE, 2003. www.nice.org.uk/pdf/TA71_coronaryarterystents_fullguidance.pdf (accessed 3 May 2005)

9 Hill R, Bagust A, Bakhai $A$, et al. Coronary artery stents: a rapid systematic review and economic evaluation. Health Technol Assess 2004;8:1-256.

10 Kiemeneij F, Serruys PW, Macaya C, et al. Continued benefit of coronary stenting versus balloon angioplasty: five-year clinical follow-up of Benestent-I trial. J Am Coll Cardiol 2001;37:1598-603.

11 Ruygrok PN, Melkert R, Morel M-AM, et al. Does angiography six months after coronary intervention influence management and outcome? J Am Coll Cardiol 1999;34:1507-11
12 Morice M-C, Serruys PW, Sousa JE, et al. A randomized comparison of a sirolimus-eluting stent with a standard stent for coronary revascularization. N Engl J Med 2002;346:1773-80.

13 Holmes DR Jr, Leon MB, Moses JW, et al. Analysis of 1-year clinical outcomes in the SIRIUS trial. Circulation 2004;109:634-40.

14 Grube E, Silber S, Hauptmann KE, et al. TAXUS I six- and twelve-month results from a randomized double-blind trial on a slow-release paclitaxel-eluting stent for de novo coronary lesions. Circulation 2003;107:38-42.

15 Colombo A, Drzewiecki J, Banning A, et al. Randomized study to assess the effectiveness of slow- and moderate-release polymer-based paclitaxel stents for coronary artery lesions. Circulation 2003;108:788-94.

16 Stone GW, Ellis SG, Cox DA, et al. One-year clinical results with the slowrelease, polymer-based, paclitaxel-eluting TAXUS stent. The TAXUS-IV trial. Circulation 2004;109:1942-7.

17 Serruys PW, Unger F, Sousa JE, et al. Comparison of coronary artery bypass surgery and stenting for the treatment of multivessel disease. N Engl J Med 2001;344:1117-24.

18 Legrand VMG, Serruys PW, Unger F, et al. Three-year outcome after coronary stenting versus bypass surgery for the treatment of multivesse disease. Circulation 2004;109:1114-20.

19 Department of Health. NHS reference costs, 2005. www.dh.gov.uk/ PolicyAndGuidance/OrganisationPolicy/FinanceAndPlanning/ NHSReferenceCosts $/ f_{s} /$ en (accessed 3 May 2005).

20 Cohen DJ, Bakhai A, Shi C, et al. Cost-effectiveness of sirolimus-eluting stents for treatment of complex coronary stenoses: results from the sirolimus-eluting balloon expandable stent in the treatment of patients with de novo native coronary artery lesions (SIRIUS) trial. Circulation 2004; 1 10:508-14.

21 The Cardiothoracic Centre Liverpool. North west quality improvement programme in cardiac interventions. www.nwheartaudit.nhs.uk (accessed 16 August 2004).

\section{IMAGES IN CARDIOLOGY}

\section{Progressive Stanford B aortic dissection treated by acute stenting}

doi: 10.1136/hrt.2005.064691

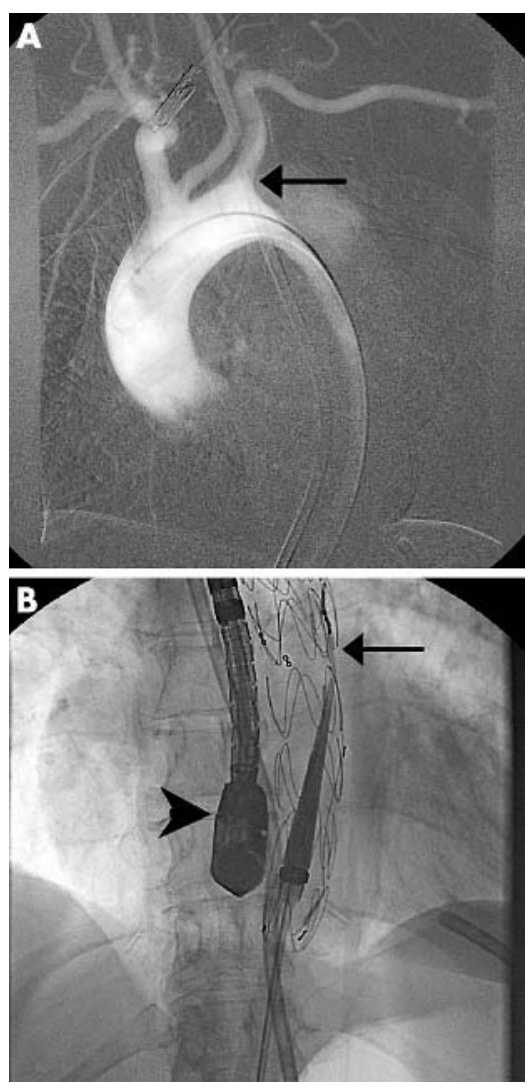

A 48 year old woman $(165 \mathrm{~cm}, 100 \mathrm{~kg})$ with a known history of untreated hypertension was admitted to our intensive care unit with severe back pain primarily located between her shoulders. Blood pressure was $250 / 120 \mathrm{~mm} \mathrm{Hg}$. Transoesophageal echocardiography showed Stanford B aortic dissection with a typical entry distal to the origin of the left subclavian artery. Contrast computed tomographic scan revealed impaired perfusion of the left kidney provided by the false lumen. The dissection ended at the height of the left iliac artery. The patient stabilised under aggressive antihypertensive treatment with a mild increase in serum creatinine and sufficient urine output. The next day she was suddenly unable to move her legs and developed anuria. The mortality risk of an open operative procedure was estimated

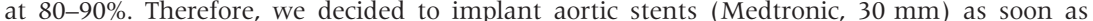
possible and intubated and ventilated the patient. Initial aortography reveals the beginning of the dissection distal to the left subclavian artery (panel A, arrow). Guided by transoesophageal echocardiography two stents were released. Radiographic control shows the result after the distal stent, which overlaps (panel B, arrow) the proximal one, has been set free. The arrowhead indicates the echocardiography probe. The first stent overlapped the origin of the left subclavian artery. The patient recovered from acute renal failure and maintained good renal function. There were no functional problems with the left arm which was perfused via retrograde flow in the left vertebral artery.

\section{J A Wagner G Wittenberg wagner_j@medizin.uni-wuerzburg.de}

